\title{
Andreev Level Qubit
}

\author{
A. Zazunov ${ }^{a}$, V. S. Shumeiko ${ }^{a}$, E. N. Bratus ${ }^{b}$, J. Lantz ${ }^{a}$, and G. Wendin ${ }^{a}$ \\ ${ }^{a}$ Department of Microelectronics and Nanoscience, Chalmers University of Technology and Göteborg University, \\ S-41296 Göteborg, Sweden \\ ${ }^{b}$ B. Verkin Institute for Low Temperature Physics and Engineering \\ 310164 Kharkov, Ukraine
}

\begin{abstract}
We investigate the dynamics of a two-level Andreev bound state system in a transmissive quantum point contact embedded in an rf-SQUID. Coherent coupling of the Andreev levels to the circulating supercurrent allows manipulation and read out of the level states. The two-level Hamiltonian for the Andreev levels is derived, and the effect of interaction with the quantum fluctuations of the induced flux is studied. We also consider an inductive coupling of qubits, and discuss the relevant SQUID parameters for qubit operation and read out.
\end{abstract}

74.80.Fp, 85.25.Dq, 03.67.Lx

Recent observations of quantum coherence in superconducting circuits [1] have made superconducting qubits a realistic possibility. Superconducting qubits employ the phenomenon of macroscopic quantum coherence (MQC) [6], and operate with coherent superpositions of quantum states of a macroscopic object - a superconducting condensate. An elementary MQC circuit consists of a hysteretic SQUID with a small capacitance Josephson tunnel junction (persistent current or flux qubit). The qubit is biased at half-integer external flux, and operates with the two degenerate current states corresponding to the clockwise and counter-clockwise circulating persistent currents. The coupling of the current states is due to quantum fluctuations of the electric charge on the junction capacitor. Quantum measurements of the fluctuating persistent current, or induced magnetic flux, provide means to read out the qubit state. Further modifications of the flux qubits involve implementation of multiple junction circuits [3, 团. In multiple junction MQC circuits, it is possible to employ one of the available dynamic variables for qubit operation, and another one for qubit readout [4] 8 ].

In this paper we consider a new type of superconducting qubit where the switching between the two persistent current states in a SQUID is achieved by employing a true microscopic system formed by the two-level Andreev bound states in a superconducting atomic-size quantum point contact embedded in the SQUID [9]. In this Andreev level qubit, the quantum information is stored in the microscopic quantum system, the Andreev bound states, similar to non-superconducting solid state qubits like localized spins on impurities 10 or quantum dots [11]. Read-out of the Andreev level qubit is achieved by monitoring the macroscopic persistent current or the induced flux in the SQUID, similar to the MQC qubits.

The Josephson effect in a single atomic-size quantum point contact (QPC) embedded in a low-inductance nonhysteretic SQUID (Fig. 1) has first been investigated by Koops et al. [12]; in this experiment, the averaged current-phase relation in the ground state was measured by performing a classical measurement of the induced flux. The results of this experiment, and also of other experiments on atomic-size QPCs where the critical current [13] and current-voltage characteristics [14.15] have been investigated, are consistent with a theoretical picture in which the Andreev bound levels play the central role in the Josephson current transport [16].

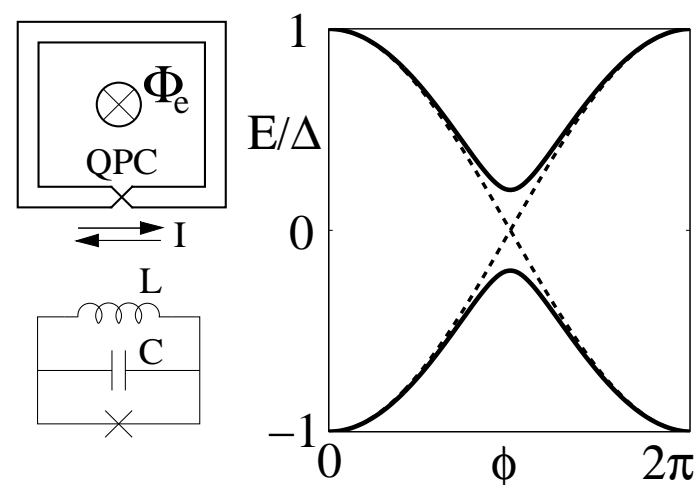

FIG. 1. Left: Sketch of the Andreev level qubit - a non-hysteretic rf-SQUID with a quantum point contact (QPC), and the equivalent circuit containing the Josephson junction and $L C$-oscillator. Right: The energy spectrum of the QPC with finite reflectivity $(R=0.04)$ (solid line), appears as a hybridization of the current states $(R=0)$ (dashed line).

The Andreev bound levels are formed in a QPC due to Andreev reflections by the discontinuity of the superconducting phase at the contact in the presence of the applied current. The Andreev level wave functions are localized in the vicinity of the contact over a distance of the order of the superconducting coherence length, and the number of Andreev bound levels is limited to one pair of levels per conducting electronic mode. Thus, a superconducting QPC may be viewed as a kind of quantum dot which contains a finite number of localized quantum states. In highly transmissive QPCs, the Andreev levels 
lie deep within the superconducting gap and are well decoupled from the continuum quasiparticle states in the electrodes.

The most important property for qubit applications is the coherent coupling of the Andreev levels to the supercurrent flowing through the contact. This makes the Andreev levels accessible for manipulations and for measurements. Extensive studies have shown that the time evolution of the Andreev levels can be controlled by applying resonant rf flux pulses [17] or by ramping the external flux [9]. By these means, one can drive Andreev levels out of the ground state and prepare any excited state [18]. On the other hand, the time evolution of the Andreev levels changes the current through the QPC. Thus the QPC operates as a quantum switch which controls the direction of the circulating current in the SQUID. Performing quantum measurement of the circulating current, or of the corresponding induced flux, in the SQUID one is able to measure the state of the Andreev level system. Fidelity of such a measurement requires the qubit evolution to be slow on a time scale of intrinsic electromagnetic fluctuations in the SQUID. Then the current (and induced flux) averaged over electromagnetic fluctuations will adiabatically follow the qubit evolution.

It is important to note that the interaction of the Andreev levels with electromagnetic modes in the SQUID is an essential element of the qubit dynamics: To maintain the current switching, the plasma frequency of the SQUID must be sufficiently large. Furtheremore, since the current in the single-mode QPC undergoes strong quantum fluctuations, the induced flux and hence the superconducting phase difference, are also fluctuating quantities, and the theory of the Andreev level qubit should include a full quantum mechanical treatment of the coupled Andreev levels and electromagnetic fluctuations.

To derive an effective quantum Hamiltonian describing coupled Andreev levels and electromagnetic fluctuations, we employ a path integral approach commonly used in MQC theory [19]. The central problem here is to extend the theory, originally developed for tunnel junctions, to the for us interesting case of high transmission QPCs; this problem is solved by using the exact boundary condition in the action instead of the tunnel Hamiltonian. Following Ref. [19], we present the evolution operator for the system on the form,

$$
U=\int \mathcal{D} \phi e^{i S_{o s c}[\phi] / \hbar} \int \mathcal{D}^{2} \psi_{L} \mathcal{D}^{2} \psi_{R} e^{i S_{J} / \hbar},
$$

where the integration is performed over the superconducting phase difference $\phi(t)$ and Nambu-Grassman fields $\psi_{L, R}(r, t)$ representing electronic degrees of freedom in the left and right electrodes respectively. The action $S_{o s c}[\phi]$ describes an $L C$-oscillator formed by the junction capacitance $C$ and the superconducting loop inductance $L$ (see Fig. 1), while the action $S_{J}$ describes the Josephson junction.

To derive the action for the Josephson junction, we consider the two superconducting reservoirs coupled via a single-channel localized scatterer. The scatterer is represented by a normal electron scattering matrix, which imposes the boundary condition for the quasiclassical wave functions of the quasiparticles in the reservoirs. The microscopic Hamiltonian for the reservoirs,

$$
H=\sum_{\sigma=L, R} \int d r \psi_{\sigma}^{\dagger} \hat{h} \psi_{\sigma}, \hat{h}=\left(\frac{\hat{p}^{2}}{2 m}-\mu\right) \sigma_{z}+\Delta \sigma_{x},
$$

is considered within the mean field approximation, the superconducting phase being gauged out and included in the boundary condition. The Hamiltonian generates the Lagrangian, $\mathcal{L}=\sum_{\sigma=L, R} \int d r \bar{\psi}_{\sigma}\left(i \hbar \partial_{t}-\hat{h}\right) \psi_{\sigma}$. In the boundary condition, which connects the fields $\psi_{L, R}(0, t)$ at the contact, the energy dependence of the scattering matrix on the scale of $\Delta$ can be neglected, so that the same matrix describes both the electrons and holes. Furthermore, without loss of generality, it is possible to eliminate constant scattering phases from the boundary condition and include them in the positive and negative momentum components of the fields $\psi_{\sigma}(r, t)$. Within the quasiclassical approximation, such a transformation will not affect the Hamiltonian in Eq. (2). The boundary condition can then be written on the form, $\chi(t)=d e^{-i \sigma_{z} \phi(t) / 2} \psi_{L}(0, t)-(1+r) \psi_{R}(0, t)=0$, where $d=\sqrt{D}$ and $r=\sqrt{R}$ are real transmission and reflection amplitudes respectively. This boundary condition is incorporated into the junction action $S_{J}$ by means of the Lagrange field $\eta(t)$,

$$
e^{i S_{J} / \hbar}=\int \mathcal{D}^{2} \eta e^{(i / \hbar) \int d t(\mathcal{L}-\bar{\eta} \chi-\bar{\chi} \eta)} .
$$

We will see later that the fermionic field $\eta(t)$ describes the Andreev levels.

Integration over the rapidly varying fermionic fields in the electrodes, $\psi_{\sigma}(r, t)$, yields the effective action

$$
e^{i S_{e f f}[\phi] / \hbar}=\int \mathcal{D}^{2} \eta e^{(i / \hbar) \int d t_{1} d t_{2} \bar{\eta}\left(t_{1}\right) G\left(t_{1}, t_{2}\right) \eta\left(t_{2}\right)},
$$

where

$$
\begin{aligned}
G\left(t_{1}, t_{2}\right)=- & \frac{D}{(1+r)^{2}} e^{-i \sigma_{z} \phi\left(t_{1}\right) / 2} g\left(t_{1}-t_{2}\right) e^{i \sigma_{z} \phi\left(t_{2}\right) / 2} \\
& -g\left(t_{1}-t_{2}\right), \quad g(\omega)=\sum_{p}(\hbar \omega-\hat{h})^{-1} .
\end{aligned}
$$

Equation (5) provides the required generalization of the the effective action of the tunnel theory [19] to junctions with arbitrary transparency. In the low frequency limit, $\omega \ll \Delta / \hbar$, the Green's function $g(\omega)$ reduces to a simple form, $g(\omega)=\left(-\pi \nu_{F} / \Delta\right)\left(\hbar \omega+\Delta \sigma_{x}\right)\left(\nu_{F}\right.$ is the density of states at the Fermi level), which leads to the local-in-time effective Lagrangian, $G\left(t_{1}, t_{2}\right)=G\left(t_{1}\right) \delta\left(t_{1}-t_{2}\right)$, 


$$
G(t)=i \hbar \partial_{t}-\frac{1-r}{4} \hbar \partial_{t} \phi \sigma_{z}+\left(\begin{array}{ll}
0 & z e^{-i \phi / 2} \\
z^{*} e^{i \phi / 2} & 0
\end{array}\right),
$$

with $z=\Delta[\cos (\phi / 2)+i r \sin (\phi / 2)]$.

Equation (6) describes a two-level fermionic system. Under stationary conditions, $\partial_{t} \phi=0$, the spectrum of the system, $\hbar \omega= \pm E_{a}, \quad E_{a}=$ $\Delta \sqrt{\cos ^{2}(\phi / 2)+R \sin ^{2}(\phi / 2)}$, coincides with the Andreev level spectrum [16], shown in Fig. 1. It follows from this equation that the assumed low-frequency approximation is appropriate for transparent contacts, $R \ll 1$, at $\phi \approx \pi$, where the Andreev level energy is small, $E_{a} \ll \Delta$. To derive the Hamiltonian for the Andreev two-level system, it is convenient first to eliminate the time derivative of the phase in Eq. (5) by means of a unitary rotation, and then to use the relation, $G(t)=i \hbar \partial_{t}-\hat{H}_{a}$, to obtain

$$
\hat{H}_{a}=\left(\begin{array}{cc}
0 & -z e^{-i r \phi / 2} \\
-z^{*} e^{i r \phi / 2} & 0
\end{array}\right) .
$$

This equation is the first main result of the present paper [20.

The slow dynamics in transparent contacts, $R \ll 1$, is described by two variables, $\eta(t)$ and $\phi(t)$. This is rather different from tunnel contacts with $D \ll 1$, where the effective action only depends on the phase difference and basically reduces to the potential Josephson energy [19]. This difference is easily understood if one takes into account that the Andreev levels in tunnel junctions are close to the gap edge, $E_{a} \approx \Delta$, making $\eta(t)$ a rapid variable. Integration over $\eta$ in Eq. (4) assuming small $D$ in Eq. (5), recovers the effective action of Ref. [19].

Let us now consider the current through the junction. The statistically averaged current $\langle I\rangle$ can be expressed through the Josephson part, $U_{J}$, of the evolution operator in Eq. (1), $\langle I\rangle=2 e i(\delta / \delta \phi) \ln \left\langle U_{J}\right\rangle$. In terms of the effective action, the equation for the averaged current reduces to the form $\langle I\rangle=\operatorname{Tr}\left(\rho_{\eta} \hat{I}\right)$, where $\rho_{\eta}$ is the density matrix, and $\hat{I}$ is the current operator of the two-level Andreev system,

$$
\hat{I}=\frac{2 e}{\hbar} \frac{d \hat{H}_{a}}{d \phi}=\frac{e \mathcal{I}(\phi)}{\hbar}\left(\begin{array}{ll}
0 & e^{-i r \phi / 2} \\
e^{i r \phi / 2} & 0
\end{array}\right),
$$

$\mathcal{I}(\phi)=\Delta D \sin (\phi / 2)$. The current operator $\hat{I}$ does not commute with the Hamiltonian $\hat{H}_{a}$, which is a consequence of the normal electron reflection at the QPC. Therefore the Andreev levels consist of superpositions of the current eigenstates, unless $R=0$, and hence the current expectation value in the Andreev state, $I_{a}=$ $(2 e / \hbar)\left(d E_{a} / d \phi\right)=(e / 2 \hbar)\left(D \Delta^{2} / E_{a}\right) \sin \phi$, does not coincide with the current eigenvalues, $\pm e \mathcal{I} / \hbar$, which are evaluated during the quantum measurements. Furthermore, the Andreev level current undergoes quantum fluctuations with the spectral function (cf. Ref. [21]),
$S(\omega)=I_{a}^{2} R \tan ^{2}(\phi / 2) \delta\left(\omega-2 E_{a}\right)$. In the SQUID geometry, these fluctuations generate strong quantum fluctuations of the phase.

We now take the quantum dynamics of the superconducting phase into the consideration. The quantum Hamiltonian of the $L C$-oscillator associated with the action $S_{o s c}[\phi]$ in Eq. (11) has the form, $\hat{H}_{o s c}=$ $-\left(\hbar \partial_{\phi}\right)^{2} / 2 M+M \omega^{2} \tilde{\phi}^{2} / 2$, where $M=\hbar^{2} / 8 E_{C}, \omega=$ $\sqrt{8 E_{L} E_{C} / \hbar^{2}}, E_{C}=e^{2} / 2 C, E_{L}=(\hbar c / 2 e)^{2}(1 / L)$, and $\tilde{\phi}$ is related to the induced flux, $\tilde{\Phi}=(c \hbar / 2 e) \tilde{\phi}$.

In the practically important case of small loop inductance, $E_{J} \ll E_{L}$, the SQUID is in the non-hysteretic regime, and the induced flux is small, $\tilde{\phi} \ll 1$. Introducing the phase difference $\phi_{e}$ related to a stationary external flux we expand the Hamiltonian in Eq. (7) over small $\tilde{\phi}$; then the Hamiltonian of the whole system in the current eigenbasis takes the form,

$$
\hat{H}=-\Delta\left(\cos \frac{\phi_{e}}{2} \sigma_{z}+r \sin \frac{\phi_{e}}{2} \sigma_{x}\right)+\frac{\mathcal{I}\left(\phi_{e}\right)}{2} \tilde{\phi} \sigma_{z}+\hat{H}_{o s c}
$$

This Hamiltonian describes a spin degree of freedom linearly coupled to an oscillator, the steady state of the oscillator being shifted from the origin by $\pm \mathcal{I} / 2 M \omega^{2}$ depending on the spin direction (direction of the current in the junction). We are interested in the case when the induced flux adiabatically follows the evolution of the Andreev levels. This regime corresponds to a large oscillator frequency compared to the Andreev level spacing, $\hbar \omega \gg 2 E_{a}$. In this case, the oscillator can be assumed to be in the ground state, $\varphi_{0}\left(\phi_{ \pm}\right), \phi_{ \pm}=\tilde{\phi} \pm \mathcal{I} / 2 M \omega^{2}$, since the probability of transitions among the oscillator levels is small. Averaging out the ground state phase fluctuations [22], we finally arrive at the effective Hamiltonian describing the Andreev level qubit,

$$
\hat{H}_{q}=-\Delta\left(\cos \frac{\phi_{e}}{2} \sigma_{z}+q_{0} r \sin \frac{\phi_{e}}{2} \sigma_{x}\right) .
$$

The factor, $q_{0}=\exp \left(-\mathcal{I}^{2} / 4 M \hbar \omega^{3}\right)$, is the overlap integral between the oscillator ground state wave functions for different current directions, $\varphi_{0}\left(\phi_{ \pm}\right)$. The averaged value of the induced phase is then given by $\langle\tilde{\phi}\rangle=\left(\mathcal{I} / 2 M \omega^{2}\right) \operatorname{Tr}\left(\rho_{\eta} \sigma_{z}\right)$, which yields the relation between the induced flux operator and the current operator, $\hat{\tilde{\Phi}}=(L / c) \hat{I}$. Therefore quantum measurement of the flux on a time scale $1 / \omega<\tau<\hbar / 2 E_{a}$ will allow correct evaluation of the Andreev level state.

The qubit Hamiltonian (10) is another main result of this paper. Equation (10) is equivalent to the Hamiltonian of non-interacting Andreev levels, Eq. (9), but with reduced reflectivity, $\tilde{R}=q_{0}^{2} R$. One may interpret this reduction as the effect of the inertia of the loop oscillator, which makes it more difficult for the Andreev levels to 
switch direction of the current. The effect becomes increasingly strong in the limit of a classical oscillator with large "mass". This renomalization effect leads to reduction of the Andreev level energy $E_{a} \rightarrow \tilde{E}_{a}$, and hence to the reduction of the frequency of the qubit rotation, remaning the results of Ref. [17,18] essentially the same. This might be important for practical applications, because it would allow the frequency of the qubit rotation to be tuned by choosing circuit parameters rather than by tuning the contact reflectivity.

The following chain of inequalities summarizes the requirements for the Andreev level qubits,

$$
2 \Delta \sqrt{\tilde{R}} \ll \hbar \omega \ll \Delta \sim E_{J} \ll E_{L} .
$$

These requirements are consistent with the typical circuit parameters of the experimental MQC qubits [3.:4. The critical current in the QPC is close to the maximum supercurrent of a single mode, $I_{c} \approx e \Delta / \hbar(\sim 400 \mathrm{nA}$ for $\mathrm{Nb}$ ), and the Josephson energy, $E_{J} \approx \Delta$. Assuming $L \sim 0.1 \mathrm{nH}$, and $C \sim 0.1 \mathrm{pF}$, we estimate $\omega \sim 10^{11} \mathrm{sec}^{-1}$, and $E_{L} / \hbar \sim 10^{13} \mathrm{sec}^{-1} \sim 10 \Delta_{N b} / \hbar$. For these values, the $q_{0}$-factor is of order unity, and the reflectivity of the contact must be small, $R \leq 0.01$, which is, in principle, accessible in the experiments with atomic-size QPC [13,15]. However, even a slight increase of the inductance will significantly decrease the $q_{0}$-factor, and the constraint on the bare contact reflectivity will become less restrictive. In summary, we estimate the upper bound for the qubit operation frequency to be about $10^{10} \mathrm{sec}^{-1}$.

The qubit operation frequency must significantly exceed the relaxation and dephasing rates of the qubit. The relaxation and dephasing mechanisms (external flux fluctuations, radiation, etc.), which have been extensively discussed for the MQC flux qubits [7], are also relevant for the Andreev level qubit. The interaction of the Andreev levels with microscopic degrees of freedom in the contact, primarily with the phonons, does not impose any further limitations. Investigation of this problem has shown 23] that the relaxation rate is very sensitive to the Andreev level spacing: for small spacing, $\tau^{-1} \sim \tilde{R}^{2} \tau_{p h}^{-1}(\Delta)$ at small temperature, $T \ll \tilde{E}_{a}$. For $\tilde{R}<0.01$ this relaxation rate is smaller than the qubit operation frequency by at least a factor of $10^{4}$.

We conclude with a discussion of the qubit-qubit interaction. Let us consider an inductive coupling as the most relevant interaction for the flux qubits. This coupling will introduce hybridization of the loop oscillators, which is described by inserting the inductance matrix in the $H_{o s c}$ term in Eq. (9). The averaging over flux fluctuations is conveniently performed using the oscillators normal modes. The averaging procedure leads to the qubit Hamiltonians in Eq. (10) with slightly different dressing factors, and to a Hamiltonian of direct qubit-qubit interaction. For the two qubits the effective interaction has the form, $H_{i n t}=-(e / c \hbar)^{2}\left(\mathcal{M I}_{1} \mathcal{I}_{2}\right) \sigma_{z 1} \sigma_{z 2}$, where
$\mathcal{M}$ is the mutual inductance. The two-qubit configuration may consist, in particular, of a single QPC with two conducting modes; in this case $\mathcal{M}=L$.

In conclusion, we have developed a theory for the Andreev level qubit, a device consisting of a SQUID with a quantum point contact, combining the features of microscopic and macroscopic quantum systems. We derived the two-level Hamiltonian for the Andreev levels and showed that it is strongly dressed by the quantum fluctuations of the induced flux. We also derived the effective interaction Hamiltonian for inductively coupled qubits, and discussed the relevant circuit parameters for the qubit operation and read out.

We acknowledge stimulating discussions with G. Johansson. This research was partially funded by project SQUBIT of the IST-FET programme of the EC.

[1] Y. Nakammura et al., Nature 398, 786 (1999).

[2] J.R. Friedman et al., Nature 406, 43 (2000).

[3] C.H. van der Wal et al., Science 290, 773 (2000).

[4] D. Vion et al., Science 296, 886 (2002).

[5] Yang Yu, et al., Science 296, 889 (2002).

[6] A. J. Leggett, in Proceedings of the NATO ASI, Les Arcs, 1983 ed. A.M. Goldman and S.A. Wolf (Plenum, New York, 1984).

[7] Y. Makhlin, et al., Rev. Mod. Phys. 73, 357 (2001).

[8] A.B. Zorin, Physica C 398, 284 (2002).

[9] J. Lantz, et al., Physica C, 368, 315 (2002).

[10] B.E. Kane, Nature 393, 133 (1998).

[11] D. Loss and D.P. DiVinchenzo, Phys. Rev. A 57, 120 (1998)

[12] M. C. Koops, et al., Phys. Rev. Lett. 77, 2542 (1996).

[13] M. F. Goffman, et al., Phys. Rev. Lett. 85, 170 (2000).

[14] N. van der Post, et al., Phys. Rev. Lett 73, 2611 (1994).

[15] E. Scheer, et al., Phys. Rev. Lett. 78, 3535 (1997).

[16] A. Furusaki and M. Tsukada, Phys. Rev. B 43, 10164 (1991); C.W. Beenakker and H. Van Houten, Phys. Rev. Lett. 66, 3056 (1991).

[17] V. S. Shumeiko, et al., Phys. Rev. B 48, 13129 (1993); L.Y. Gorelik, et al., Phys. Rev. Lett. 75, 1162 (1995).

[18] V.S. Shumeiko, E.N. Bratus', and G. Wendin, in Proceedings of the XXXII Moriond Conference, Les Arcs, 2001, ed. T. Martin, G. Montambaux, J. Tran Thanh Van (IDP Sciences, 2001).

[19] V. Ambegaokar, et al., Phys. Rev. Lett. 48, 1745 (1982); U. Eckern, et al., Phys. Rev. B 30, 6419 (1984).

[20] Truncation of the Bogoliubov-de Gennes equation 18] gives the same Hamiltonian; Eq. (8) is different from the one presented in literature (e.g., D. A. Ivanov and M. V. Feigelman, Phys. Rev. B 598444 (1998)).

[21] A. Martin-Rodero, et al., Phys. Rev. B 53, R8891 (1996).

[22] A.J. Leggett, et al., Rev. Mod. Phys. 59, 1 (1987).

[23] D. A. Ivanov and M. V. Feigelman, JETP Lett. 68, 890 (1998). 